\title{
Schedule-induced wood-chewing in rats and its dependence on body weight
}

\author{
T. J. ROPER and G. CROSSLAND \\ University of Sussex, Brighton, Sussex BNI $9 Q G$, England
}

\begin{abstract}
In Experiment 1, 12 rats were exposed to an FT 60 schedule of food reinforcement, followed either by extinction or by a massed-food control condition, in the presence of a wood block. In 9 rats, wood-chewing behavior increased systematically during the FT 60 condition and declined again during extinction or massed food, while the other 3 rats showed virtually no chewing behavior at any stage of the experiment. In Experiment 2, frequency and bout duration of wood-chewing under an FT 60 schedule of food reinforcement declined as body weight increased, in 7 rats. We conclude that wood-chewing qualifies as a schedule-induced behavior, and that it resembles schedule-induced drinking in its dependence on body weight. Unlike drinking, however, induced chewing occupied the middle region of the 60-sec interreinforcement interval, declined markedly within the session, and showed considerable within- and between-subject variability.
\end{abstract}

Falk (1961) found that when rats were allowed to obtain food according to an intermittent (VI 60-sec) schedule of food reinforcement, and were given concurrent free access to a water bottle, they developed the habit of drinking immediately after eating each food pellet. Because the rats' cumulative intake of water under these circumstances was abnormally large, Falk referred to the postpellet drinking as "schedule-induced polydipsia." Schedule-induced polydipsia has been extensively studied in the subsequent two decades, but its cause remains obscure: numerous explanations have been offered, but none has proved satisfactory (see reviews by Falk, 1971; Segal, 1972; Staddon, 1977).

Explanations of schedule-induced drinking have been of two distinct types, which may be termed "thirst" explanations and "general activation" explanations, respectively (Roper, 1980b). "Thirst" explanations hold that intermittent presentation of food exerts a specific facilitatory effect on drinking, for example by stimulating oral dehydration receptors (Stein, 1964) or by causing a fall in blood glucose level (Freed, Zec, \& Mendelson, 1977). "General activation" explanations, on the other hand, hold that intermittent presentation of food exerts a more or less general facilitatory effect on nonfeeding behaviors, via a state or process termed frustration (e.g., Thomka \& Rosellini, 1975), arousal (e.g., Killeen, 1975; Brett \& Levine, 1979), general motor excitability (Wayner, 1974), or stress (e.g., Wallace \& Singer, 1976).

At present most investigators seem to favor a gen-

Financial support was provided by the U.K. Science Research Council. Reprints may be obtained from: T. J. Roper, School of Biology, University of Sussex, Brighton, Sussex BN1 9QG, England. eral activation account, on the grounds that schedule induction extends across a variety of species, reinforcers, and behaviors. But whether one regards induction as a general phenomenon depends on the stringency of one's definition. The term "scheduleinduced" is commonly applied to any activity that occurs during interreinforcement intervals, regardless of its frequency of occurrence, whereas a stringent demonstration of induction requires that the behavior in question be shown to occur more frequently in association with a schedule of reinforcement than in a nonschedule baseline condition or conditions. More specifically, Roper (1981) suggests that a behavior should be termed "schedule-induced" only if its frequency of occurrence under an intermittent schedule of reinforcement be shown to exceed its frequency of occurrence (1) under a no-reinforcer or extinction condition, and (2) under a massed-reinforcer condition.

So far, drinking in rats, and a few other species, and aggression in pigeons are the only behaviors that have been shown to be schedule-induced by this dualbaseline criterion; and for aggression, the evidence is somewhat equivocal (see review by Roper, 1981). Thus, although there is reason to suppose that scheduleinduced drinking is fairly general across species, there is little evidence that induction extends to activities other than drinking, and no evidence that it is produced by reinforcers other than food.

The present study demonstrates the occurrence of schedule-induced wood-chewing in rats with food as reinforcer, by comparison with extinction and massedfood baseline conditions; and it shows that induced chewing, like drinking, varies as a function of the rat's body weight. Wood-chewing has previously been reported in rats in conjunction with intermittent 
schedules of food reinforcement (Laties, Weiss, \& Weiss, 1969; Killeen, 1975; Roper, 1978), but its frequency of occurrence has not previously been assessed in nonschedule baseline conditions.

\section{EXPERIMENT 1}

An unambiguous demonstration of schedule induction might use an ABA design, in which $A$ is a nonschedule baseline condition (no-food or massedfood) and B is an intermittent schedule of reinforcement. However, we have found that initial exposure of rats to wood in a nonschedule condition prevents the subsequent appearance of induced chewing (unpublished observations), making an ABA design impossible. In Experiment 1, therefore, there was no initial baseline condition: one group of rats was exposed first to FI 60 and then to extinction, while another was exposed first to FI 60 and then to a massed-food condition.

\section{Method}

Subjects. Twelve naive female hooded rats (mean weight $242 \mathrm{~g}$, range 223 to $276 \mathrm{~g}$ ) were obtained from the laboratory colony. They were housed individually under a reversed light cycle (light on 2000 to $0800 \mathrm{~h}$ ) and were maintained at $80 \%$ of free-feeding body weight.

Apparatus. The rats were tested in four identical operant chambers. Each chamber measured $24 \times 22 \times 20 \mathrm{~cm}$, and contained a response lever, a food tray, and a "chewometer." The response lever $(3.8 \times 1.8 \times 1.4 \mathrm{~cm}$, Campden Instruments $L$ tdd. $)$ and the recessed food tray $(5.3 \times 6.0 \times 3.5 \mathrm{~cm}$, Campden Instruments Ltd.) were mounted $10 \mathrm{~cm}$ apart on the same wall, and food pellets (Campden Instruments Ltd. complete diet formula, $45 \mathrm{mg}$ ) were delivered to the tray by an automatic dispenser. The lever operated a switch when depressed with a force of $.15 \mathrm{~N}$. The from of the food tray was covered by a hinged Plexiglas flap $(5.3 \times$ $6.0 \mathrm{~cm}$ ) which operated a microswitch when pushed open, allowing collection of food pellets to be monitored automatically. A stimulus light was mounted above the food tray.

Mounted on the opposite wall was a "chewometer" consisting of a block of oak $(4 \times 3 \times 2 \mathrm{~cm})$ bolted onto the face of a pigeon key (Campden Instruments Ltd.) so as to protrude into the chamber at 2 height of $4 \mathrm{~cm}$. A brass counterweight was bolted to the rear of the key. Horizontal or vertical pressure on the oak block caused the pigeon key to swing back, operating a switch. Observations made during this study and a previous one (Roper, 1978) verified that rats were unable to chew the wood block without operating the switch and that the switch was rarely operated during other types of behavior.

Each chamber was housed in a sound-attenuating wooden chest, fitted with a one-way observation window and located in an airconditioned testing room. Activities were monitored and schedules were controlled by apparatus located in an adjacent room.

Procedure. On Day 1, each rat was placed in an operant chamber for $60 \mathrm{~min}$, with the food-tray flap wedged open and 60 pellets in the tray. On Day 2, a pellet was delivered each time the rat pushed open the food-tray flap, and on Day 3 the response of leverpressing was shaped. On Days 4 and 5, leverpressing was reinforced according to an FR 1 schedule, delivery of a pellet being signaled by 2.5 -sec illumination of the food-tray light. On Days 6 to 15 , leverpressing was reinforced according to a fixed-interval 60-sec (FI 60) schedule. All sessions lasted for $60 \mathrm{~min}$, and during pretraining no wood was present in the test chamber.

The experiment proper consisted of two phases. During the first phase (20 sessions) leverpressing continued to be reinforced ac- cording to an FI 60 schedule, but a wood block was made concurrently available in each chamber. During the second phase (10 sessions), six rats (designated Group A) experienced an extinction condition in which no food was available. The other six rats (Group B) experienced a massed-food condition in which leverpressing again had no scheduled consequences, but in which 60 pellets were placed in the food tray prior to the start of the session.

Leverpresses, food-tray responses and wood-block displacements were recorded automatically. Video facilities were used periodically during the experiment to verify that wood-block displacements accurately reflected chewing behavior.

\section{Results}

Figures 1 and 2 show the number of leverpresses per session and the total chew time per session for each rat in the last five sessions of pretraining and in all subsequent sessions. ("Chew time" refers to the total duration of chewometer switch closures, and is a less variable measure of chewing than the absolute number of switch closures.) Three rats (A1, B2, and B5) showed virtually no wood-chewing behavior at any stage of the experiment. In the other nine rats, chew time increased during the FI 60 (wood present) condition and declined again during extinction (Group A) or massed-food (Group B) conditions. Damage to the oak blocks and direct observation of the behavior confirmed that vigorous woodchewing was occurring in the FI 60 condition in nine rats, but Figures 1 and 2 show considerable individual and day-to-day variation in chewing scores.

Considering now the leverpressing scores, Figures 1 and 2 show that introduction of the wood block

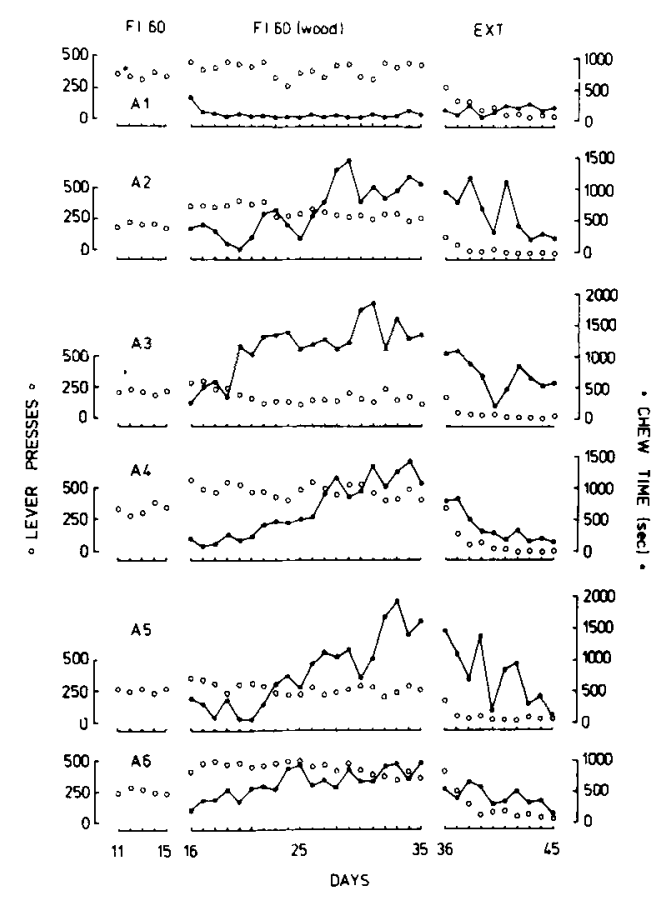

Figure 1. Number of leverpresses per session (open circles) and chew time per session (filled circles) for the six rats in Group A, during the last five sessions of pretraining and all subsequent sessions. 
caused, in most rats, an increase in number of presses per session. During the FI 60 (wood present) condition, leverpressing scores remained relatively constant, suggesting that wood-chewing behavior did not compete significantly with operant behavior. During the extinction and massed-food conditions, leverpressing declined rapidly in all rats.

Figure 3 shows the temporal distribution of woodchewing and leverpressing during the interreinforce-
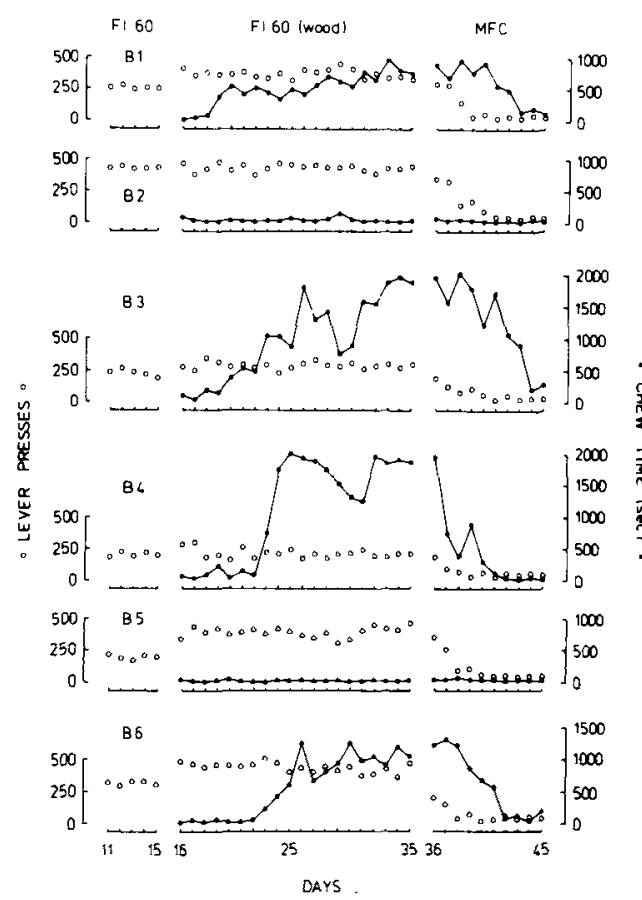

Figure 2. Number of leverpresses per session (open circles) and chew time per session (filled circles) for the six rats in Group B, during the last five sessions of pretraining and all subsequent sessions.

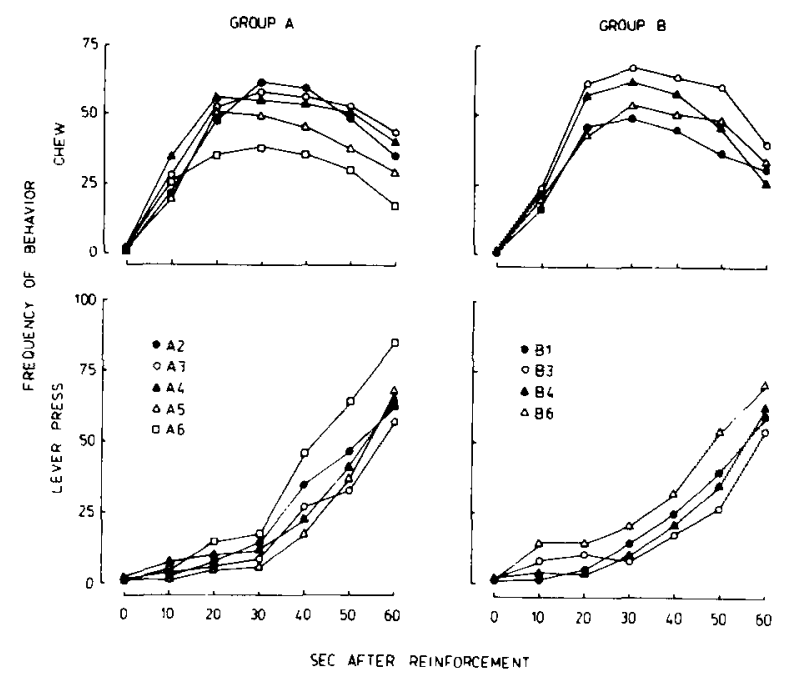

Figure 3. Frequency of occurrence of chewing (upper panels) and leverpressing (lower panels) as a function of time since reinforcement. Left-hand panels refer to the five rats in Group A; right-band panels refer to the four rats in Group $B$.

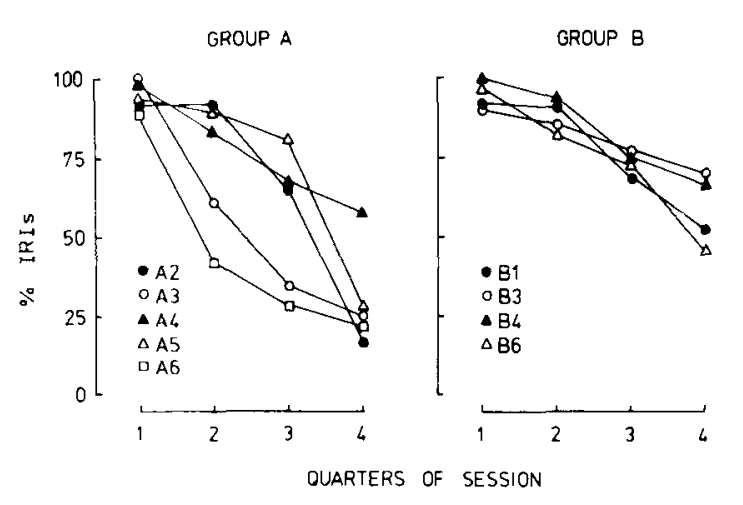

Figure 4. Percentage of interreinforcement intervals (IRIs) contoining wood-chewing in successive quarters of the session. The left-hand panel refers to the five rats in Group $A$; the right-hand panel refers to the four rats in Group $B$.

ment interval in the nine rats that showed significant chewing behavior. Data were taken from the last session of the FI 60 (wood present) condition. The figure shows the frequency of each behavior at successive 10 -sec intervals following delivery of food. The left-hand graphs show the results for rats in Group A; the right-hand graphs refer to Group B. In all rats, wood-chewing behavior peaked about halfway through the 60-sec interreinforcement interval, while leverpressing increased in an exponential manner towards the end of the interval.

Figure 4 shows the distribution of wood-chewing within the last session of the FI 60 (wood present) condition for the nine rats that engaged in significant chewing behavior. In successive quarters of the session, the graphs show the percentage of interreinforcement intervals in which chewing occurred. There was a clear within-session decline in the frequency of chewing in all rats.

\section{Discussion}

Five of the six rats in Group A engaged in more wood-chewing under an FI 60 schedule of food reinforcement than in a subsequent extinction condition, while four of six rats in Group B engaged in more chewing under FI 60 than in a subsequent massedfood condition. The results therefore show that woodchewing complies with the dual-baseline criterion of schedule-induction suggested by Roper (1981), at least in the majority of rats.

On the other hand, the results suggest a number of differences between schedule-induced chewing and induced drinking. First, induced chewing was less reliable in several respects than induced drinking: it failed to occur in some subjects, was slow to develop, showed considerable day-to-day variability, and declined sharply within the session. Second, chewing occurred with maximum frequency in the middle region of the 60 -sec interreinforcement interval (see, also, Roper, 1978), whereas drinking typically 
occurs immediately after the consumption of a food pellet (for references see Staddon, 1977).

Experiment 2 was intended to pursue the analogy between induced chewing and induced drinking by investigating the effect of body weight on induced chewing. Several studies (Falk, 1969; Freed \& Hymowitz, 1972; Roper \& Nieto, 1979) have shown that, in rats, induced drinking declines both in frequency and in bout duration as body weight increases.

\section{EXPERIMENT 2}

Experiments on induced drinking have used two methods of controlling body weight: Falk (1969) allowed the weights of his rats to vary gradually between $80 \%$ and $105 \%$ of free-feeding level, while Freed and Hymowitz (1972) and Roper and Nieto (1979) held the weights of their rats steady at different levels until asymptotic rates of induced drinking were achieved. In Experiment 2, the former procedure was used with one group of rats and the latter procedure with another group.

\section{Method}

Subjects and Apparatus. The subjects were seven of the nine rats that had shown induced chewing in Experiment 1 (Rat A6 was rejected because it failed to engage in chewing behavior when an FI 60 schedule was reimposed following Experiment 1, and Rat B3 was rejected because of an ear infection). The apparatus was as in Experiment 1.

Procedure. Following Experiment 1, the rats received 15 sessions under an FI 60 schedule of food reinforcement, with wood present. By the end of this period, wood-chewing appeared to have reached asymptotic level.

The FI 60 schedule was continued throughout the experiment, with 1-h sessions. The four rats in Group A were tested for three sessions at $80 \%$ body weight, following which their body weights were allowed to increase gradually. When body weights had reached $110 \%$ of initial free-feeding value, or when chewing had declined to a low level, body weights were returned gradually to the $80 \%$ level.

The three rats in Group B experienced seven sessions at each of the following body weights: $80 \%, 90 \%, 100 \%$, and $80 \%$.

\section{Results}

Figure 5 shows the changes in wood-chewing scores as a function of body weight, for the four rats in Group A. Three measures of chewing are shown: the total time spent chewing per session, the percentage of interreinforcement intervals in which chewing occurred, and the average duration of chewing per interreinforcement interval ("bout duration'). The three measures of chewing declined in all four rats as body weight increased, but were subject to considerable day-to-day variability. Scores tended to be higher in the weight-loss phase of the experiment (open circles in Figure 5), especially in Rat AS.

Figure 6 shows the same three scores (total chew time per session, percentage of interreinforcement intervals containing chewing, and average bout duration of chewing) for the three rats in Group B. Filled
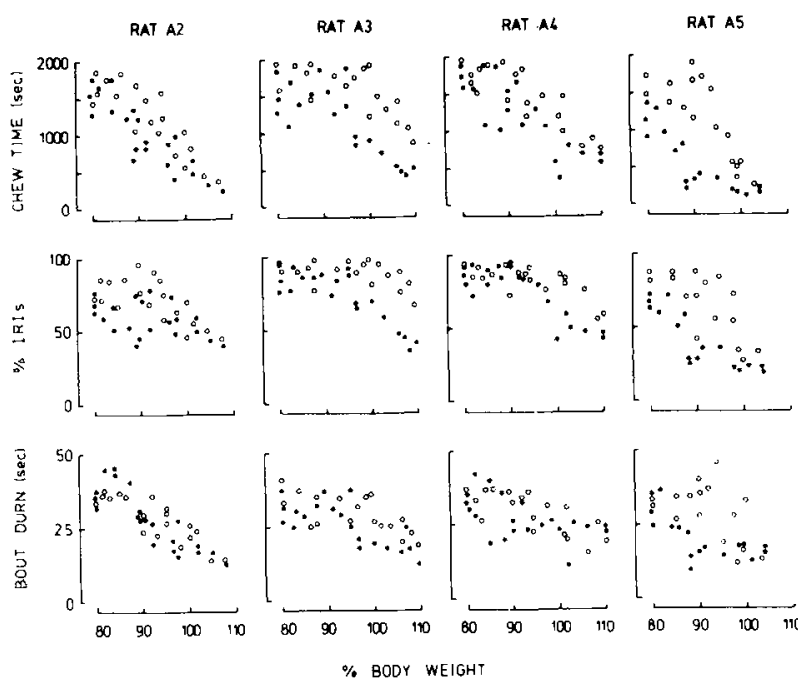

Figure 5. Chew time per session (upper panels), percentage of interreinforcement intervals containing chewing (central panels), and average bout duration of chewing (lower panels) as a function of percentage body weight, for the four rats in Group A. Filled circles = descending body weight; open circles = ascending body weight.

circles show the scores for each of the last three sessions at a particular body weight, while histogram bars show the average score over these 3 days. Chew time, percentage intervals, and bout duration all declined as body weight increased in all three rats, and the scores recovered again when body weight was returned to the $80 \%$ level.

Although there was some decline in rate of leverpressing as body weight increased, this was less marked than the decline in chewing, and did not result in reduction in the number of reinforcements obtained per session. Records of food-tray entries showed that
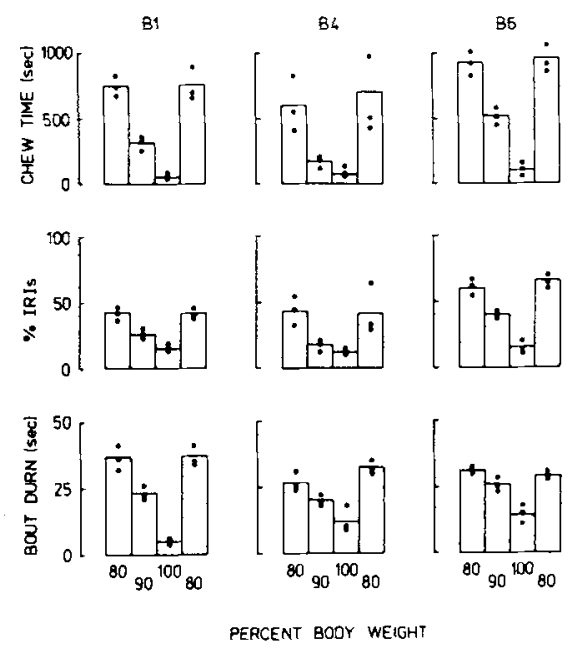

Figure 6. Chew time per session (upper panels), percentage of interreinforcement intervals containing chewing (central panels), and average bout duration of chewing (lower panels) as a function of percentage body weight, for the three rats in Group B. Each point represents data from a single session; histogram bar length represents the mean over three sessions. 
rats continued to collect, with short latency, all the food pellets that they obtained, even at body weights exceeding the initial $100 \%$ level.

\section{Discussion}

Previous studies of schedule-induced drinking (Falk, 1969; Freed \& Hymowitz, 1972; Roper \& Nieto, 1979) have shown that the amount drunk per session, the frequency with which drinking follows reinforcement, and the average bout duration of drinking all decline as the rat's body weight increases towards free-feeding value. Experiment 2 showed a decline in the same three measures of induced wood-chewing, showing at least a qualitative similarity between the two types of induced behavior.

Quantitatively, however, there may be differences in the extent to which induced drinking and chewing are affected by body weight. In a study directly comparable to Experiment 2, Roper and Nieto (1979) found that induced drinking continued at a substantial level at $100 \%$ body weight, albeit drinking was reduced by comparison with $80 \%$ weight. In Experiment 2 , by contrast, chewing was virtually nonexistent at $100 \%$ body weight.

\section{GENERAL DISCUSSION}

Two main points emerge from the present study. First, wood-chewing complies with the criterion of schedule induction suggested by Roper (1981): that is, the amount of chewing under an intermittent (in this case, FI 60) schedule of reinforcement exceeds the amount of chewing both in extinction and in a massedfood condition. The only other behaviors that have so far been shown to comply with Roper's criterion of schedule induction are drinking in rats and primates and aggression in pigeons (for references, see Roper, 1981).

Second, the present study showed a decline in the frequency and bout duration of wood-chewing as body weight approached free-feeding value. Scheduleinduced drinking in rats (Falk, 1969; Freed \& Hymowitz, 1972; Roper \& Nieto, 1979) and aggression in pigeons (Dove, 1976) vary in much the same way, as a function of body weight.

Although these results suggest basic similarities between induced chewing, drinking, and aggression, these three types of behavior seem to differ in a number of other respects. First, by comparison with induced drinking, the induced chewing observed in Experiment 1 was slow to appear, showed considerable individual and day-to-day variability, and declined markedly within the session. Slow acquisition and individual variability also seem to be characteristic of induced aggression (e.g., Looney \& Cohen, 1978, report that aggression can take up to 30 sessions to appear and that it fails to occur in a substantial pro- portion of birds); but there have been no reports of a within-session decline in aggression.

Second, there is a marked difference between the temporal distribution within the interreinforcement interval of chewing, on the one hand, and drinking and aggression, on the other. Thus, chewing tends to peak about halfway through a 60 -sec interreinforcement interval (Figure 3 and Roper, 1978), whereas drinking and aggression typically (though not invariably) occur early in the interval, immediately after eating (for references, see Staddon, 1977).

Third, we have found that induced chewing fails to appear if rats are preexposed to wood during nonschedule baseline conditions (Roper \& Crossland, Note 1). Preexposure to baseline conditions does not affect the occurrence of induced drinking (e.g., Roper \& Posadas-Andrews, 1981; Roper, 1980a), but there is some evidence that it retards or prevents the appearance of induced aggression (Looney, Cohen, \& Yoburn, 1976; Looney \& Dove, 1978). Presumably, preexposure to nonschedule baseline conditions results in habituation to the chewing- or aggressionprovoking stimulus.

Fourth, unpublished observations suggest that the occurrence of chewing is strongly dependent, in two respects, on the stimulus characteristics of the wood block; hard woods are greatly preferred to soft woods, and the introduction of a new block of wood typically causes a temporary increase in chewing. The latter effect, together with the fact that chewing is prevented by preexposure to wood (see above), suggests that stimulus novelty is an important factor. Stimulus novelty could also explain the waning of chewing within the session, although it is possible that this is caused by a decline in the reinforcement value of food due to satiation. Induced aggression also varies according to the nature of the precise stimulus used to elicit it (e.g., Flory \& Ellis, 1973; Looney \& Cohen, 1974; Macurik, Kohn, \& Kavanaugh, 1978), whereas induced drinking seems to be relatively independent of the stimulus characteristics of the fluid available for consumption. Thus, rats can be induced to drink a wide variety of substances, including some, such as alcohol, that would normally be rejected (e.g., Riley, Lotter, \& Kulkosky, 1979).

In conclusion, induced drinking, chewing, and aggression seem to resemble one another in some respects but to differ in others. What are the implications for accounts of schedule induction? The fact that chewing is schedule-induced supports the popular, but hitherto poorly substantiated, view that activities other than drinking are susceptible to induction. Consequently, the results tend to favor an explanation of induction in terms of some general process such as arousal or frustration, rather than in terms of a specific process of "thirst" (cf. Roper, 1981). The demonstration that induced chewing de- 
clines as body weight increases is also consistent with a frustration or arousal account, because intermittent delivery of food would be expected to be more frustrating or arousing, the hungrier the animal. On the other hand, not all the activities in a species' repertoire seem to be capable of induction: there is evidence that wheel-running and grooming cannot be induced by intermittent schedules of reinforcement in rats (Penney \& Schull, 1977; Roper, 1980b; Roper \& Nieto, 1979; Wetherington, Brownstein, \& Schull, 1977). Thus, important questions remain concerning the readiness with which different activities can be induced, and these questions can only be answered by systematic examination of a wide range of potential induced activities. Similarly, the apparent differences in the detailed properties of induced chewing, drinking, and aggression suggest that unitary explanations of induction in terms of a single variable may be oversimplified.

More specifically, the results militate against one particular account of schedule induction, namely that of Staddon (1977). According to Staddon, schedule-induced behavior is a response to low probability of reinforcement, from which it follows that induced behavior should be most frequent immediately after the consumption of food. The present results are directly contrary to this prediction, because they show schedule induction of chewing despite the fact that chewing occurs in the middle of the interreinforcement interval. Staddon's account is also undermined by the fact that induced drinking will occur in any part of the interreinforcement interval if water availability is restricted to that part (e.g., Gilbert, 1974). An alternative approach to the question of temporal patterning of interreinforcement behavior has been offered by Roper (1980b), who suggests that the sequencing of interreinforcement activities merely reflects the sequencing of the same activities in the rat's normal ongoing behavior. According to this view, the question of where an activity is preferentially located in the interreinforcement interval bears no relationship to the question of whether that activity is susceptible to schedule induction.

One final question concerns the nature of the woodchewing behavior observed in the present experiment. It is commonly assumed that frustration causes aggression (e.g., Hutchinson, Azrin, \& Hunt, 1968), and there have been numerous demonstrations of schedule-induced aggression against a conspecific in pigeons. Furthermore, there is some evidence that biting an inanimate object, or jaw-clenching, is correlated with unambiguously aggressive behavior in rats, monkeys, and humans (Hutchinson, Pierce, Emley, Proni, \& Sauer, 1977; Hutchinson \& Renfrew, 1978). It is therefore tempting to suppose that scheduleinduced wood-chewing is aggressive in nature, and certainly our rats grappled with the wood and gnawed it in an extraordinarily vigorous manner. However, some caution is necessary on this point, because attempts to obtain schedule-induced aggression against a conspecific target in rats have so far proved unsuccessful (Gentry \& Schaeffer, 1969; Hymowitz, 1971; Knutson \& Schrader, 1975).

\section{REFERENCE NOTE}

1. Roper, T. J., \& Crossland, G. Unpublished findings, 1980.

\section{REFERENCES}

Bretr, L. D., \& Levine, S. Schedule-induced polydipsia suppresses pituitary-adrenal activity in rats. Journal of Comparative and Physiological Psychology, 1979, 93, 946-956.

Dove, L. D. Relation between level of food deprivation and rate of schedule-induced attack. Journal of the Experimental Analysis of Behavior, 1976, 25, 63-68.

FALK, J. L. Production of polydipsia in normal rats by an intermittent food schedule. Science, 1961, 133, 195-196.

FALK, J. L. Conditions producing psychogenic polydipsia in animals. Annals of the New York Academy of Science, 1969, 157, 569-593.

FALK, J. L. The nature and determinants of adjunctive behavior. Physiology \& Behavior, 1971, 6, 577-588.

FLony, R. K., \& ElL1s, B. B. Schedule-induced aggression against a slide-image target. Bulletin of the Psychonomic Society, $1973,2,287-289$.

Freed, E. X., \& Hyмowitz, N. Effects of schedule, percent body weight, and magnitude of reinforcer on acquisition of schedule-induced polydipsia. Psychological Reports, 1972, 31, 95-101:

Freed, W. J., Zec, R. F., \& Mendelson, J, Schedule-induced polydipsia: The role of orolingual factors and a new hypothesis. In J. A. W. M. Weijnen \& J. Mendelson (Eds.), Drinking behavior: Oral stimulation, reinforcement and preference. New York: Plenum, 1977.

Gentry, W. D., \& Schaeffer, R. W. The effect of FR response requirement on aggressive behavior in rats. Psychonomic Science, $1969,14,236-238$.

GILBERT, R. M. Ubiquity of schedule-induced polydipsia. Journal of the Experimental Analysis of Behavior, 1974, 21, 277-284.

Hutchinson, R. R., Azrin, N. H., \& Hunt, G. M. Attack produced by intermittent reinforcement of a concurrent operant response. Journal of the Experimental Analysis of Behavior, $1968,11,489-495$.

Hutchinson, R. R., Pierce, G. E., Emley, G. S., Proni, T. J., \& Sauen, R. A. The laboratory measurement of human anger. Biobehavioral Reviews, 1977, 1, 241-259.

Hutchinson, R. R., \& Renfrew, J. W. Functional parallels between the neural and environmental antecedents of aggression. Neuroscience \& Biobehavioral Reviews, 1978, 2, 33-58.

Hyмоwiтz, N. Schedule-induced polydipsia and aggression in rats. Psychonomic Science, 1971, 23, 226-228.

Killesw, P. On the temporal control of behavior. Psychological Review, 1975, 82, 89-115.

Knutson, J. F., \& Schrader, S. P. A concurtent assessment of schedule-induced aggression and schedule-induced polydipsia in the rat. Animal Learning \& Behavior, 1975, 3, 16-20.

Laties, V. G., Weiss, B., \& Weiss, A. B. Further observations on overt "mediating" behavior and the discrimination of time. Journal of the Experimental Analysis of Behavior, 1969, 12, 47-57.

Looney, T. A., \& Cohen, P. S. Pictorial target control of schedule-induced attack in White Carneaux pigeons. Journal of the Experimental Analysis of Behavior, 1974, 21, 571-584. Looney, T, A., \& Cohen, L. D. Schedule-induced attack as a 
function of length of exposure to a fixed-time $90-\mathrm{sec}$ schedule. Bulletin of the Psychonomic Society, 1978, 12, 320-322.

Looney, T. A., Cohen, P. S., \& Yobunn, B. C. Variables affecting establishment of schedule-induced attack on pictorial targets in White King pigeons. Journal of the Experimental Analysis of Behavior, 1976, 26, 349-360.

LoONEY, T. A., \& Dove, L. D. Schedule-induced attack as a function of length of exposure to a fixed-time 90 -sec schedule. Bulletin of the Psychonomic Society, 1978, 12, 320-322.

Macurik, K. M., Kohn, J. P., \& Kavanaugh, E. An alternative target in the study of schedule-induced aggression in pigeons. Journal of the Experimental Analysis of Behavior, 1978, 29. 337-339.

Penney, J., \& Schuld, J. Functional differentiation of adjunctive drinking and wheel running in rats. Animal Learning \& Behavior, 1977, 5, 272-280.

Riley, A. L., Lotter, E. C., \& Kulkosky, P. J. The effects of conditional taste aversions on the acquisition and maintenance of schedule-induced polydipsia. Animal Learning \& Behavior, $1979,7,3-12$.

ROPER, T. J. Diversity and substitutability of adjunctive activities under fixed-interval schedules of food reinforcement. Journal of the Experimental Analysis of Behavior, 1978, 30, 83-96.

ROPER, T. J. Changes in rate of schedule-induced behaviour in rats as a function of fixed-interval schedule. Quarterly Journal of Experimental Psychology, 1980, 32, 159-170. (a)

ROPER. T. J. Behaviour of rats during self-initiated pauses in feeding and drinking, and during periodic response-independent delivery of food and water. Quarterly Journal of Experimental Psychology, 1980, 32, 459-472. (b)

ROPER, T. J. What is meant by the term "schedule-induced," and how general is schedule induction? Animal Learning \& Behavior, $1981,9,433-440$.

Rope R, T. J., \& NiETo, J. Schedule-induced drinking and other behavior in the rat, as a function of body-weight deficit. Physiology \& Behavior, 1979, 23, 673-678.

Roper, T. J., \& Posadas-Andrews, A. Are schedule-induced drinking and displacement activities causally related? Quarterly Journal of Experimental Psychology, 1981, 33B, 181-193.

SEgaL, E. F. Induction and the provenance of operants. In R. M. Gilbert \& J. R. Millenson (Eds.), Reinforcement: Behavioral analyses, New York: Academic Press, 1972.

Staddon, J. E. R. Schedule-induced behavior. In W. K. Honig \& J. E. R. Staddon (Eds.), Handbook of operant behavior. New York: Prentice-Hall, 1977.

Ste IN, L. Excessive drinking in the rat: Superstition or thirst? Journal of Comparative and Physiological Psychology, 1964, 58, 237-242.

Thомка, M. L., \& Rosellini, R. A. Frustration and the production of schedule-induced polydipsia. Animal Learning \& Behavior, 1975, 3, 380-384.

Wallace, M., \& Singer, G. Schedule-induced behavior: A review of its generality, determinants and pharmacological data. Pharmacology, Biochemistry \& Behavior, 1976, 5, 483-490.

WAyner, M. J. Specificity of behavioral regulation. Physiology \& Behavior, 1974, 12, 851-869.

Wetherington, C. L., Brownstein, A. J., \& Shull, R. L. Schedule-induced running and chamber size. Psychological Record, 1977, 27, 703-713.

(Manuscript received May 26, 1981; revision accepted for publication September 8, 1981.) 